\title{
Psicooncología
}

ISSN: $1696-7240$

http://dx.doi.org/10.5209/PSIC.55819

\section{Pronosticar con fechas ¿es la mejor manera de informar?}

Anna Rodríguez-Morera*

Recibido: 20 de febrero de 2017 / Aceptado: 15 de abril de 2017

Sumario. 1. Referencias bibliográficas.

Cómo citar: Rodríguez-Morera. Pronosticar con fechas ¿es la mejor manera de informar? Psicooncología 2017; 14: 159-61. Doi:10.5209/PSIC.55819

La estimación del pronóstico forma parte de una habilidad fundamental para la buena práctica clínica, de ella dependen muchas decisiones médicas y el uso eficiente de los recursos sanitarios. Asimismo, de una estimación errónea se pueden derivar consecuencias negativas para el paciente y su familia. No obstante diversos factores la convierten en inevitablemente inexacta a la vez que fácilmente condicionada por la experiencia del profesional y el tipo de relación mantenida entre médico y paciente $^{(1)}$.

Algunos estudios anglosajones observan una disminución significativa del malestar ante la información detallada del pronóstico ${ }^{(2)}$ mas en nuestra población no tenemos evidencia concluyente de que el conocimiento del pronóstico per se produzca un beneficio psicológico directo al paciente con cáncer ${ }^{(3)}$.

La mayoría de médicos están convencidos de que se tiene que facilitar toda la información del pronóstico al paciente pero en realidad no lo hacen, lo hacen parcialmente o solo lo hacen en aquellos casos en que el paciente lo pide explícitamente ${ }^{(4)} \mathrm{o}$ cuando necesitan la autorización expresa del paciente para algún tratamiento, prueba o documento ${ }^{(5)}$.

Cuando la enfermedad avanza la familia acostumbra a necesitar más información y más concreta sobre la expectativa de supervivencia pero el paciente menos y más centrada en el control de síntomas ${ }^{(6)} \mathrm{o}$ incluso puede desear no recibir más información médica ${ }^{(5)}$. Curiosamente bastantes pacientes informados no aceptan el pronóstico de su enfermedad y buscan maneras alternativas neutras de interpretar la situación para convertirla en más llevadera ${ }^{(6-8)}$. También en aquellos casos que preguntan explícitamente y afirman querer saber la verdad pueden preferir quedar parcialmente ignorantes de su expectativa de $\operatorname{vida}^{(6,8)}$.

Conociendo la imprecisión que pueden tener los pronósticos cerrados ${ }^{(1)}$ y el dudoso beneficio que pueden tener para el paciente ${ }^{(3)}$ ¿Por qué seguimos poniendo fechas

* Dirección de correspondencia: Hospital Universitari de Girona Dr. Josep Trueta. Facultat de Medicina. Universitat de Girona. E-mail: amorera@iconcologia.net 
exactas en las expectativas de vida de los pacientes? El proceso de información se ha puesto a disposición de los servicios sanitarios y de sus necesidades, no a disposición de las necesidades del paciente y de sus familiares.

El médico busca legalidad, ciencia y realismo, pero el paciente busca poder vivir a pesar de su enfermedad y necesita paradójicamente una situación ambigua con una mezcla de honestidad y de esperanza ${ }^{(3,8)}$. Se presupone que los pacientes quieren preparar sus cosas, despedirse de los suyos, arreglar malentendidos, preparar su manera de morir... en definitiva, que el autocontrol y la consciencia de final de vida le ayudaran en su bienestar y calidad de vida ${ }^{(7)}$ pero ¿en realidad, es esto siempre así? Posiblemente no.

La moda de poner fechas cerradas de pronóstico (por ejemplo: le queda un mes, unos tres meses, menos de seis meses, un año, más de un año...), además de poder ser inexactas y poco honestas, ocasionan impacto en las esperanzas del paciente y de su familia dejando poco espacio para el maquillaje adaptativo que necesita hacer el paciente de su realidad ${ }^{(4)}$. La esperanza, o a mi entender, la ilusión de poder cambiar las cosas que van mal, tiene un matiz distinto en cada persona ${ }^{(9)}$ y no siempre se tiene que vestir en términos médicos ${ }^{(4)}$. El paciente tiene derecho a imaginar mejoras, a esperar cambios físicos, a querer detener su enfermedad, a creer que puede curarse... mientras va elaborando el propio proceso de aceptación del posible final de vida cercano ${ }^{(4)}$.

¿Puede pues el oncólogo informar de forma honesta y mantener la esperanza del paciente a la vez? Claro que si. En definitiva no se trata de si hay una manera correcta o incorrecta de informar, si no de ofrecer el "pronóstico soportable”, que no es más que proponer al paciente la mejor información posible sobre su enfermedad en cada momento concreto ${ }^{(10)}$.

\section{Referencias bibliográficas}

1. Maltoni M, Caraceni A, Brunelli C, Broeckaert B, Christakis N, Eychmueller S, et al. Steering Committee of the European Association for Palliative Care. Prognostic factors in advanced cancer patients: evidence-based clinical recommendations-a study by the Steering Committee of the European Association for Palliative Care. J Clin Oncol 2005;23:6240-8. Doi: 10.1200/JCO.2005.06866

2. Lundquist $\mathrm{G}$, Rasmussen BH, Axelsson B. Information of imminent death or not: does it make a difference? J Clin Oncol 2011 Oct 10;29:3927-31. Doi: 10.1200/ JCO.2011.34.6247. Epub 2011 Sep 12.

3. Nuñez Olarte JM. Información al paciente en situación terminal: diferencias entre las publicaciones anglosajonas y la experiencia diaria en España. Med Pal 2014; 21:113-20. Doi:10.1016/j.medipa.2012.11.004

4. BackAL,Anderson WG, Bunch L, Marr LA, Wallace JA, Yang HB,ArnoldRM. Communication about cancer near the end of life. Cancer 2008;113:1897-910. Doi:10.1002/cncr.23653

5. Cuesta J, Martínez A, Rodriguez A. Informar o no informar a los pacientes paliativos: ¿qué opinan sus familiares? Med Pal 2012;19;58-63. Doi: 10.1016/j.medipa.2010.10.001

6. Hagerty RG, Butow PN, Ellis PA, Lobb EA, Pendlebury S, Leighl N, Goldstein D, Lo SK, Tattersall MH. Cancer patient preferences for communication of prognosis in the metastatic setting. J Clin Oncol 2004;22:1721-30. Doi: 10.1200/JCO.2004.04.095 
7. Weeks JC, Catalano, PJ, Cronin, Finkelman MD, Mack JW, Keating NL y Schrag D. Patients' expectations about effects of chemotherapy for advanced cancer. N Engl J Med 2012;367:1616-25. Doi: 10.1056/NEJMoa1204410

8. Innes S, Payne S. Advanced cancer patient? Prognostic information preferences: a review. Palliat Med 2009; 23:29-39. Doi: 10.1177/0269216308098799 Epub 2008 oct 24

9. Clayton JM, Hancock K, Parker S, Butow PN, Walder S, Carrick S, Currow D, Ghersi D, Glare P, Hagerty R, Olver IN, Tattersall MH. Sustaining hope when communicating with terminally ill patients and their families: a systematic review. Psychooncology 2008;17: 641-59. Doi: 10.1002/pon. 1288

10. Antequera JM, Barbero J, Bátiz J, Bayés R, Casares M, Gérvas J, et al. Guías de ética en la práctica médica. Ética en cuidados paliativos. Fundación de Ciencias de la Salud. Madrid : Ergon; 2006. 